\title{
Lipopolysaccharide-induced changes in the neurovascular unit in the preterm fetal sheep brain
}

\author{
Clémence Disdier', Fares Awa'1, Xiaodi Chen', Simerdeep K. Dhillon², Robert Galinsky², Joanne O. Davidson², \\ Christopher A. Lear ${ }^{2}$, Laura Bennet ${ }^{2}$, Alistair J. Gunn ${ }^{2}$ and Barbara S. Stonestreet ${ }^{{ }^{*}}$ (D)
}

\begin{abstract}
Background: Exposure to inflammation during pregnancy can predispose to brain injury in premature infants. In the present study, we investigated the effects of prolonged exposure to inflammation on the cerebrovasculature of preterm fetal sheep.

Methods: Chronically instrumented fetal sheep at 103-104 days of gestation (full term is $\sim 147$ days) received continuous low-dose lipopolysaccharide (LPS) infusions (100 ng/kg over $24 \mathrm{~h}$, followed by $250 \mathrm{ng} / \mathrm{kg} / 24 \mathrm{~h}$ for $96 \mathrm{~h}$ plus boluses of $1 \mu \mathrm{g}$ LPS at 48, 72 , and $96 \mathrm{~h}$ ) or the same volume of normal saline $(0.9 \%, \mathrm{w} / \mathrm{v})$. Ten days after the start of LPS exposure at 113-114 days of gestation, the sheep were killed, and the fetal brain perfused with formalin in situ. Vessel density, pericyte and astrocyte coverage of the blood vessels, and astrogliosis in the cerebral cortex and white matter were determined using immunohistochemistry.

Results: LPS exposure reduced $(P<0.05)$ microvascular vessel density and pericyte vascular coverage in the cerebral cortex and white matter of preterm fetal sheep, and increased the activation of perivascular astrocytes, but decreased astrocytic vessel coverage in the white matter.

Conclusions: Prolonged exposure to LPS in preterm fetal sheep resulted in decreased vessel density and neurovascular remodeling, suggesting that chronic inflammation adversely affects the neurovascular unit and, therefore, could contribute to long-term impairment of brain development.
\end{abstract}

Keywords: Cortex, Fetal brain, Inflammation, Lipopolysaccharide, Neurovascular unit, Sheep, White matter

\section{Background}

Exposure to inflammation and/or infectious agents resulting from premature rupture of membranes and chorioamnionitis during gestation is highly associated with adverse neonatal sequela including chronic lung disease, adverse neurodevelopmental outcomes along with long-term disabilities [1-3]. Premature infants

\footnotetext{
*Correspondence: bstonestreet@wihri.org

1 Department of Pediatrics, Women \& Infants Hospital of Rhode Island, Alpert Medical School of Brown University, 101 Dudley Street, Providence, RI 02905, USA

Full list of author information is available at the end of the article
}

surviving after exposure to inflammation around the time of birth are at high risk for a wide spectrum of adverse neurodevelopmental outcomes, presumably, because of injury to the developing brain [1-7]. Moreover, they are at continued risk for disability throughout childhood [6-9]. Lipopolysaccharide (LPS) is a gramnegative bacterial endotoxin that is widely used as a robust inflammatory catalyst to stimulate neuroinflammatory responses in a variety of animal models including fetal sheep [10-15].

A complex network of blood vessels is required to provide nutrients and oxygen to the developing brain. The

(C) The Author(s). 2020 Open Access This article is licensed under a Creative Commons Attribution 4.0 International License, which permits use, sharing, adaptation, distribution and reproduction in any medium or format, as long as you give appropriate credit to the original author(s) and the source, provide a link to the Creative Commons licence, and indicate if changes were made. The images or other third party material in this article are included in the article's Creative Commons licence, unless indicated otherwise in a credit line to the material. If material is not included in the article's Creative Commons licence and your intended use is not permitted by statutory regulation or exceeds the permitted use, you will need to obtain permission directly from the copyright holder. To view a copy of this licence, visit http://creativecommons.org/licenses/by/4.0/ The Creative Commons Public Domain Dedication waiver (http://creativecommons.org/publicdomain/zero/1.0/) applies to the data made available in this article, unless otherwise stated in a credit line to the data. 
neurovascular unit (NVU) integrates cell interactions between brain endothelial cells, the basal lamina, and the surrounding glial limitans and pericytes [16]. Each component of the NVU is intimately interconnected with the other constituents to establish an effective anatomical and functional system [17]. This unique complex system maintains brain homeostasis, preserves the integrity of the blood-brain barrier (BBB), protects the brain from toxic endogenous and exogenous substances, regulates blood flow, and provides growth factors needed to support proper neuronal development. The barrier mechanisms of the human, rat, and sheep brain are present from very early in gestation [18-23]. The fetal and neonatal BBB are effective from early in development and possesses many characteristics of the barrier observed in the fully developed adult brain [18-23].

Inflammation has long been known to promote BBB disruption and cerebrovascular dysfunction [24-27]. Inflammatory signals and neurovascular dysfunction can both result in neuronal damage $[25,28,29]$. Nonetheless, the effects of inflammation on the response of the NVU in the immature brain remain to be determined. Lipopolysaccharide (LPS) is a gram-negative bacterial endotoxin that is widely used as a robust inflammatory catalyst to stimulate neuroinflammatory responses in a variety of animal models including fetal sheep [10-15]. Single intravenous doses of LPS administered to fetal sheep resulted in extravasation of albumin into the brain parenchyma suggesting BBB disruption [30]. Similarly, injection of LPS into the uterine artery of pregnant ewes resulted in extravasation of plasma albumin into the cerebellar parenchyma of the fetus suggesting that maternal exposure to LPS also can compromise the fetal BBB [31]. Furthermore, exposure to LPS during development alters capillary density and interferes with angiogenesis in microvasculature of the retina, which is a vasculature bed that is structurally similar to the $\mathrm{BBB}$ $[32,33]$. Taken together, these findings suggest that the neurovasculature in premature subjects could be vulnerable to inflammatory insults.

The overall goal of the current study was to test the hypothesis that prolonged exposure to inflammation adversely affects the neurovasculature in the brain of preterm fetal sheep. Therefore, we examined the effects of a prolonged exposure to a regimen of LPS on the quantity of blood vessels in the brain and changes in the NVU in fetal sheep in order to simulate exposure to in utero inflammation.

\section{Methods}

\section{Animal preparation, study groups, and experimental design}

All procedures were approved by the Animal Ethics Committee of the University of Auckland and carried out in accordance with the New Zealand Animal Welfare Act, and the Code of Ethical Conduct for animals in research established by the Ministry of Primary Industries, Government of New Zealand. The tissue samples for this study were obtained from animals, in which physiological changes and white matter injury have been previously reported $[14,15,34,35]$.

\section{Fetal surgery}

Singleton Romney/Suffolk fetal sheep were surgically instrumented at 98-100 days of gestation (term $=147$ days) as previously reported for the purpose of the original studies [14, 15, 34, 35]. The ewes were given long-acting oxytetracycline $(20 \mathrm{mg} / \mathrm{kg}$, Phoenix Pharm, Auckland, New Zealand) intramuscularly $30 \mathrm{~min}$ before the onset of surgery. Anesthesia was induced by an intravenous injection of propofol $(5 \mathrm{mg} / \mathrm{kg}$; AstraZeneca Limited, Auckland, New Zealand) and maintained using $2-3 \%$ isoflurane in oxygen (Bomac Animal Health, NSW, Australia). During surgery, ewes received an intravenous infusion of normal $(0.9 \%, \mathrm{w} / \mathrm{v})$ saline $(250 \mathrm{~mL} / \mathrm{h})$ to maintain an appropriate fluid balance. The depth of anesthesia, maternal heart rate, and respiration were continuously monitored by trained anesthetic staff.

A midline incision was made to expose the uterus, and the fetus was partially exteriorized for instrumentation. Polyvinyl catheters were placed in the left femoral artery and amniotic sac for the original studies. Catheters were also placed in the right brachial artery and left femoral vein for the intravenous infusions of LPS and normal $(0.9 \%, \mathrm{w} / \mathrm{v})$ saline.

All fetal catheters were exteriorized through the maternal flank, and a maternal saphenous vein was catheterized for post-operative care and euthanasia. Antibiotics were administered into the amniotic sac (gentamicin, $80 \mathrm{mg}$, Pharmacia and Upjohn, Rydalmere, NSW, Australia) before the uterus was closed. Ewes were given $5 \mathrm{~mL}$ of Streptocin (procaine penicillin, 250,000 IU/mL, and dihydrostreptomycin, $250 \mathrm{mg} / \mathrm{mL}$, Stockguard Labs, Hamilton, New Zealand) intramuscularly $30 \mathrm{~min}$ before surgery for prophylaxis. The maternal midline skin incision was infiltrated with local analgesic $(10 \mathrm{~mL} 0.5 \%$ bupivacaine plus adrenaline, AstraZeneca Ltd., Auckland, New Zealand).

\section{Post-operative care}

After surgery, ewes were housed together in separate metabolic cages with ad libitum access to food and water. Rooms were temperature and humidity controlled $\left(16 \pm 1{ }^{\circ} \mathrm{C}\right.$, humidity $\left.50 \pm 10 \%\right)$ with a 12 -h light/dark cycle (light 06:00 to 18:00 h). Ewes were given daily intravenous antibiotics $(600 \mathrm{mg}$ Crystapen, Biochemie, Vienna, Austria and Gentamicin, $80 \mathrm{mg}$, for 4 days after surgery. Fetal catheters were maintained patent with 
continuous infusions of heparinized normal $(0.9 \%, \mathrm{w} / \mathrm{v}$; $20 \mathrm{U} / \mathrm{mL}$ at $0.2 \mathrm{~mL} / \mathrm{h}$ ).

\section{Experimental protocol}

The experiments commenced 5 days after recovery from surgery at 103-104 days of gestation. This time in gestation is approximately comparable neuroanatomically to that of the preterm human brain between 28 and 32 weeks of gestation [36]. It represents $70 \%$ of the ovine gestation because full-term gestation in this breed of fetal sheep is 147 days. Hereafter, we refer to this time in gestation as $70 \%$ of gestation. Figure 1a shows a schema of the study design. Fetuses were randomly assigned to either (1) chronic normal saline infusions and saline boluses (saline controls, $n=8,3$ females and 5 males) and (2) chronic LPS (055:B5, Sigma Aldrich, St. Louis, MO, USA) infusion and LPS boluses $(n=7,2$ females and 5 males). LPS was dissolved in normal saline and infused at $100 \mathrm{ng} / \mathrm{kg}(50 \mathrm{ng} / \mathrm{mL}$ at $83 \mu \mathrm{L} / \mathrm{h})$ for the first $24 \mathrm{~h}$ followed by $250 \mathrm{ng} / \mathrm{kg} / 24 \mathrm{~h}(50 \mathrm{ng} / \mathrm{mL}$ at $207.5 \mu \mathrm{L} / \mathrm{h})$ for the next $96 \mathrm{~h}$. Boluses were administered as $1 \mu \mathrm{g}$ LPS dissolved in $1 \mathrm{~mL}$ of normal saline at 48, 72, and $96 \mathrm{~h}$ from the start of infusion. Normal saline controls received equivalent volumes of saline for both the infusions and boluses. This LPS regimen was intended to produce a prolonged exposure to the inflammatory stimulus $[14,15,34,35]$ to simulate conditions similar to those in human pregnancies with prolonged rupture of membranes and chorioamnionitis and also to limit LPS-related fetal demise $[37,38]$. Ten days after the start of the infusions at 113-114 days of gestation, the ewe was euthanized with an overdose of pentobarbital sodium (Pentobarb 300, Chemstock international, Christchurch, New Zealand). The fetal brain was perfused with heparinized normal saline (20 IU heparin $/ 500 \mathrm{ml}$ saline) followed by $1 \mathrm{~L}$ of $10 \%$ neutral buffered formalin. The brains were fixed for an additional 7 days in 10\% neutral buffered formalin and divided into 4 to $5 \mathrm{~mm}$ thick coronal sections using a sheep brain slicer matrix and embedded in paraffin.

\section{Immunochemical staining and microscopy}

Immunohistochemical analysis was performed on $10 \mu \mathrm{m}$ deparaffinized and rehydrated coronal brain sections. Sections were prepared for glial fibrillary acidic protein (GFAP) and desmin plus collagen type IV double immunofluorescence as follows. The brain sections were immersed in EDTA buffer (1 mM EDTA, Sigma Aldrich, $0.05 \%$ Tween 20, Sigma Aldrich; $\mathrm{pH}=8$ ) and heated for $15 \mathrm{~min}$ in a pressure cooker in a microwave. The slides were rinsed with Tris-buffered saline (TBS, $10 \mathrm{mM}$, Sigma Aldrich) containing 0.025\% triton-X100 (TBS-T, Sigma Aldrich) twice for $5 \mathrm{~min}$ each after the heatinduced epitope retrieval. The brain sections were then blocked in SuperBlock T20 (TBS) blocking buffer for $2 \mathrm{~h}$ at room temperature. The two primary antibodies were incubated overnight on two sequential days. First, the anti-GFAP (1:1000 dilution, Invitrogen, Carlsbad, CA, USA) or the anti-desmin (1:100 dilution, Invitrogen) were incubated overnight at $4{ }^{\circ} \mathrm{C}$. On the second day, the slides were rinsed twice in TBS for $5 \mathrm{~min}$ and then the sections were incubated with collagen type IV antibody (1:100 dilution, Origen, Rockville, MD, USA) at 4 ${ }^{\circ} \mathrm{C}$ overnight to visualize the brain microvessels. On the next day, the slides were rinsed with TBS-T twice for 5 min, and then TBS and incubated with the appropriate Alexa Fluor 594- and 488-conjugated secondary antibodies (1:1000 dilution, Invitrogen) for $1 \mathrm{~h}$ in the dark at room temperature. The slides were then rinsed with TBS-T twice for $5 \mathrm{~min}$ then TBS and sections were subsequently cover slipped with DAPI containing mounting medium (Vector Laboratories, Burlingame, CA, USA). Staining for microglia was performed on sections that were immersed in citrate buffer $(0.1 \mathrm{M}$ citrate buffer, $\mathrm{pH} 6$ ) and heated for $15 \mathrm{~min}$ in a pressure cooker to achieve antigen retrieval. The sections were then rinsed in TBS-T and blocked for $2 \mathrm{~h}$ in SuperBlock T20 (TBS) blocking buffer (Thermo Scientific, Wilmington, DE, USA). They were then incubated with anti-ionized calcium-binding adaptor molecule 1 (Iba-1) antibody (1: 1000 dilution, Invitrogen) at $4{ }^{\circ} \mathrm{C}$ overnight. Thereafter, the slides were rinsed and incubated with Alexa Fluor 594-conjugated secondary antibodies (1:1000 dilution, Invitrogen) and then mounted as described above. Immunostaining without primary antibodies were used to rule out non-specific binding. All images were digitized and visualized using a Zeiss Axio Imager M2 Imaging System microscope (Carl Zeiss, Inc., Jena, Germany). The image acquisitions and analyses were performed on coded slides by two examiners (C.D. and F.A.), who were masked to the groups. Each investigator undertook the analysis separately. The final data represent the average values from both investigators. Simple adjustments were made to each entire image for the purpose of image presentation including adjustments in the contrast and intensity histogram strictly according to published guidelines [39].

\section{Quantification of single-labeled immunofluorescence}

One brain section per animal was randomly selected for imaging and analysis. Fifteen to 20 fields per section were randomly selected for imaging and analysis based upon the collagen type IV staining of the blood vessels for the single-labeled immunohistochemical quantification of collagen type IV and GFAP in the cerebral cortical and white matter regions. The fields were systematically sampled in the cerebral cortical and white matter regions of the preterm fetal sheep brain such that 


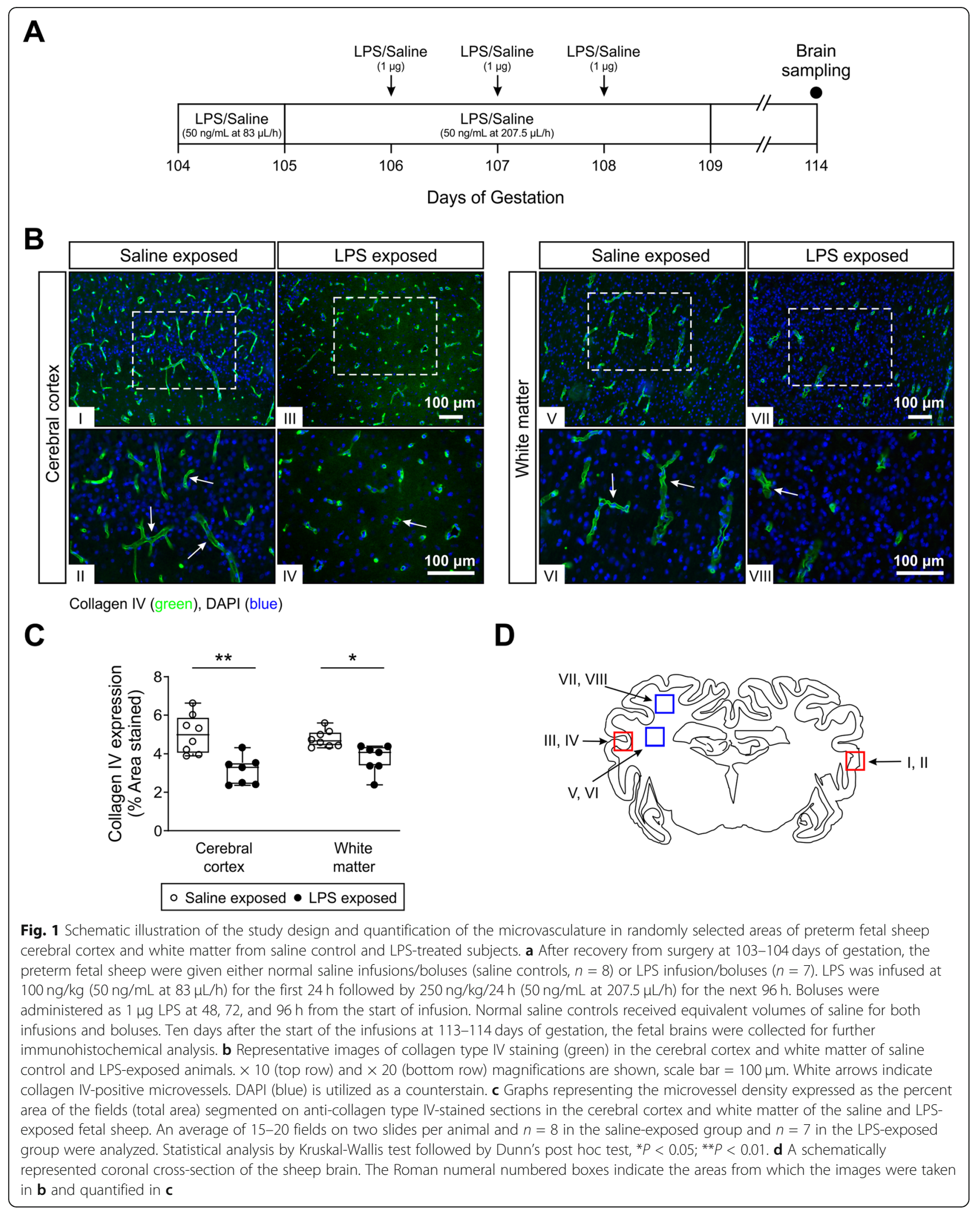


an average area of $50 \mu \mathrm{m}^{2}$ and $44 \mu \mathrm{m}^{2}$ were sampled for cerebral cortex and white matter, respectively. Low and high power images were taken at $\times 10$ and $\times 20$, respectively. Images were segmented using an interactive thresholding modality, and the resulting binary images were processed with the measurement function of ImageJ software (NIH, Bethesda, MD). The immunoreactive positive areas were measured and quantified, and the final analysis expressed as an area fraction of the total area examined, which is the percent of the total field area that had been highlighted in green for collagen type IV or red for GFAP. Only blood vessels larger than $50 \mu \mathrm{m}$ were used for the purpose of analysis.

\section{Quantification of pericyte and astrocytic blood vessel coverage}

Multichannel channel images were acquired in order to quantify the blood vessel coverage by astrocytes and pericytes. Randomly selected vessels were imaged at $x$ 40 magnification in the cerebral cortex or white matter based upon the collagen type IV staining. The channels were then split into the channels for the GFAP (red) or desmin (red) channels and the collagen IV (green) channel. The collagen IV source images were then thresholded to define the outer margin of the blood vessel under consideration and to measure the total blood vessel area. The red channel (GFAP or desmin) was then subtracted from the collagen IV (green) channel using the ImageJ image calculator tool. The same threshold used for the collagen IV was used for the resulting image and the area overlapping the red for the astrocytes or pericytes over the green blood vessel area was determined. The vascular coverage for the astrocytes and pericytes was expressed as a percentage of the total blood vessel surface area.

Quantitative image analysis was performed by two independent observers (C.D. and F.A.), who were not aware of the of the group designations. The two observers demonstrated excellent agreement for the astrocytic and pericytic microvascular coverage, $r=0.89$, $n=15, P<0.0001$ and $r=0.95, n=15, P<0.0001$, respectively. The final results represented the average of the mean values by the two independent observers. One brain section per animal and 15-20 fields randomly selected fields per section were analyzed in the cerebral cortex and white matter for all experiments in each brain of the fetuses from the control $(n=8)$ and LPS ( $n$ $=7$ ) treated fetal sheep for each protein of interest.

\section{Statistical analysis}

Results are presented in the text as mean and standard deviation of the mean. Box-and-whisker plots depict the median, 10th, 25th, 75th, and 90th percentiles as vertical boxes with error bars. Statistical analyses were performed using Graphpad Prism ${ }^{\circ}$ (GraphPad Software, Inc, San Diego CA). Kruskal-Wallis test followed by Dunn's post hoc test was used to compare the differences between the two groups. Linear regression analyses were used to determine correlations between GFAP and the vessel density; astrocytic coverage and the vessel density and pericyte coverage and the vessel density. $F$ test was used to calculate the $P$ values for the linear regression analyses. Differences were considered statistically significant if $P<0.05$.

\section{Results}

Exposure to LPS reduces microvascular vessel density in the preterm fetal brain

Collagen type IV is a major molecular component of the vascular basal lamina, which we have previously demonstrated to be an excellent marker of the microvasculature in the fetal sheep brain [40]. Therefore, collagen type IV staining was used to visualize the microvascular network in the preterm fetal sheep cerebral cortex and white matter 10 days after the start of exposure to saline or LPS (Fig. 1). The number of collagen type IV green stained microvessels were greater in the cerebral cortex and white matter of the saline (Fig. 1b) than the LPS exposed fetal sheep. Analytic quantification of collagen type IV staining at low magnification (Fig. 1c) revealed a reduction in the quantity of the collagen type IVimmunostained microvessels in the cerebral cortex and white matter of the LPS compared with the saline exposed control fetal sheep. The percentage area stained with collagen IV in the cerebral cortex was $3.13 \pm 0.73 \%$ in the LPS compared with $5.02 \pm 1.00 \%$ in the saline exposed group. Similarly, the microvessel density was also lower in the white matter of the LPS, $3.7 \pm 0.7 \%$, compared with the saline, $4.8 \pm 0.4 \%$, exposed fetal sheep. The cross area of the sheep brain is schematically represented in Fig. 1d. The boxes designated by Roman numerals indicate the areas from which the images were obtained in Fig. 1b and quantified for the graphical analysis in Fig. 1c.

\section{Exposure to LPS reduces pericyte and vascular smooth muscle microvascular blood vessel coverage in the preterm fetal sheep brain}

Desmin is a muscle-specific class III intermediate filament found in pericytes and vascular smooth muscle cells that ensheath the microvasculature [41]. Pericyte and vascular smooth muscle cell coverage of the microvessels were examined by double immunostaining for desmin and collagen type IV in the cerebral cortical and white matter regions of the fetal sheep brain (Fig. 2a, b). The pericyte and vascular smooth muscle desmin coverage of the microvessels was significantly lower in the 


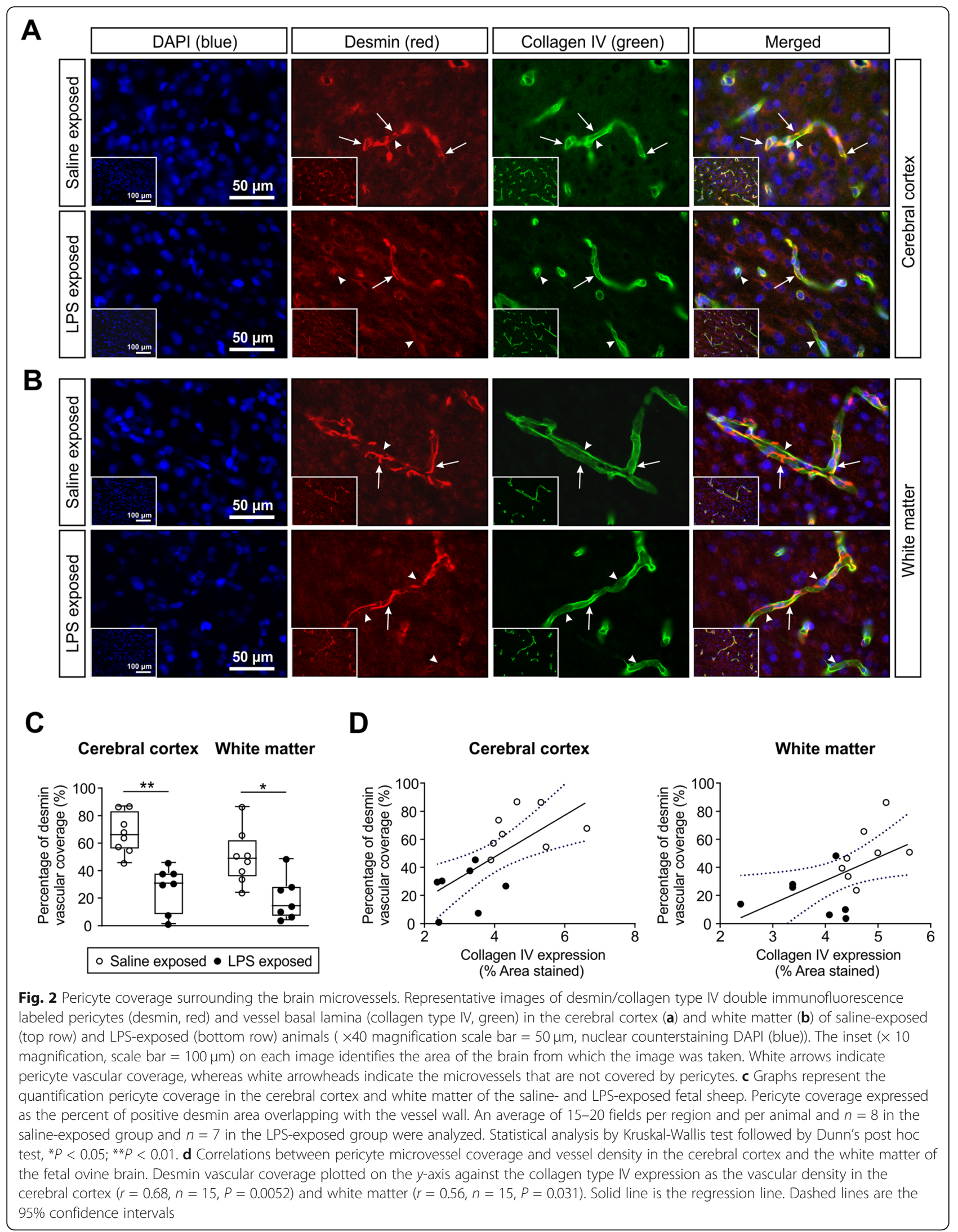


cerebral cortex and white matter of LPS compared with saline-exposed fetal sheep (Fig. 2a, b).

In the saline-exposed control group, we observed extensive desmin staining of the vascular walls corresponding to $66.9 \pm 14.8 \%$ of the vascular coverage in the cerebral cortex and $49.5 \pm 19.4 \%$ in the white matter (Fig. 2c). In the LPS exposed group, we observed significant reductions in the pericyte coverage corresponding to $40 \%$ in both cerebral cortex and white matter regions (Fig. 2c). In addition, we found a significant correlation between the desmin pericyte coverage and microvessel density in both the cerebral cortex $(r=0.68, n=15, P=$ 0.005, Fig. 2d) and white matter $(r=0.56, n=15, P=$ 0.031 , Fig. $2 \mathrm{~d}$ ) supporting the concept of interrelated deficiencies in the constituents of the neurovascular unit.

\section{Exposure to LPS results in astrocyte activation and reduces astrocytic microvessel coverage in the white matter}

Double immunolabeling was performed with anti-GFAP as an astrocytic marker and anti-collagen type IV as a component of the basal lamina in both groups. The antiGFAP antibody stained the astrocyte cell bodies, processes, and end-feet equally (Figs. 3a and 4a). Astrocytes exhibited a typical fine morphology with GFAP reactive processes that formed the end-feet surrounding the blood vessels in the saline-exposed fetal sheep brains (Figs. 3a and 4a). Astrocytes were stained in both the cerebral cortex and white matter. LPS exposure was associated with reactive type astrocytic morphology characterized by hypertrophy and swelling of the astrocyte body and processes (Figs. 3a and 4a). Exposure to the LPS regimen resulted in the activation of the astrocytes in the white matter (Figs. 3a and 4a). The morphological changes were not associated with significant increases in GFAP immunoreactivity in the cerebral cortex. The average cerebral cortical GFAP fluorescence per unit area was $19.3 \pm 6.6$ in the saline compared with $24.4 \pm$ 6.4 in the LPS-exposed group (Fig. 3b). GFAP immunoreactivity in the white matter was significantly greater in the LPS compared with the saline-exposed group suggesting the presence of reactive astrogliosis. The GFAP average fluorescence per unit area in the white matter was $16.4 \pm 3.9$ in the saline versus $26.1 \pm 3.5$ in the LPSexposed group (Fig. 3b). The GFAP expression per unit area showed an inverse correlation with collagen type IV expression in the white matter $(r=0.58, n=15, p=$ 0.022 , Fig. 3c), but not in the cerebral cortex (Fig. 3c).

The overlap of GFAP and collagen type IV was quantified to evaluate astrocyte end-feet coverage surrounding the brain microvessels (Fig. 4). As expected in the salineexposed group, astrocyte end-feet were present surrounding the brain microvessels within the cerebral cortex and white matter, indicating close contact between the glia limitans and the microvasculature (Fig. 4a). The average astrocytic coverage in the saline group was $51.7 \pm 14.9 \%$ of the vascular area in the cerebral cortex and $48.8 \pm 10.5 \%$ in the white matter (Fig. $4 \mathrm{~b}$ ). The development of hypertrophic end-foot processes was associated with significant decreases in the overlap of GFAP and collagen type IV in the white matter of the LPS-exposed group, $26.8 \pm 13.4 \%$ of the vascular area, but not in the cerebral cortex, $37.1 \pm 14.3 \%$ of the vascular area in the cortex. This suggests that exposure to LPS diminished the close contacts between the vasculature and astrocytes surrounding the microvessels in the white matter (Fig. 4b). In addition, we demonstrated a direct correlation between the astrocyte vascular coverage and the vascular density $(r=0.69, n=15, P=$ 0.0041 , Fig. 4c) in the white matter. However, a significant correlation was not observed in the cerebral cortex for the same analysis (Fig. 4c).

\section{Exposure to LPS results in microglial activation}

The LPS-related inflammatory signals predispose to increases in BBB permeability and facilitate the entry of macrophages and cytokines from the blood into the brain to further accentuate neuroinflammation [42-45]. We used Iba-1 as a marker in this study because it recognizes the ramified cellular shape of the microglia. The resting-type microglia were characterized in the salineexposed control fetal sheep by a ramified phenotype evenly distributed in the perivascular space of the cerebral cortex and white matter (Supplemental Figure 1A and B upper panel). We confirmed the presence of activated amoeboid microglia in perivascular spaces in the white matter of LPS exposed animals (Supplemental Figure 1B lower panel). By contrast, there were no morphological changes of microglia in the cerebral cortex of LPS exposed fetal sheep (Supplemental Figure 1A lower panel) consistent with the previous report in the same animal model [14].

In the current study, we also attempted to co-label the microvasculature along with microglia in order to quantify the microglia that were associated with the microvasculature. However, even after attempting to stain the microvessels with collagen type IV along with the microglia using four different antibodies (anti-Iba1 antibody, chicken polyclonal 139590, Abcam, Cambridge, MA, USA; anti-Iba1 antibody, mouse monoclonal 15690, Abcam, anti-Iba1 antibody, mouse monoclonal, 01626721 FUJIFILM Wako, Richmond, VA, USA; and antiIba1 antibody, goat polyclonal, ab5076, Abcam) and several different heat antigen retrieval buffers, we were not able to obtain reliable results simultaneously with the two stains in the fetal sheep brain tissue. Therefore, we were unable to quantify microglia associated with microvessels. 


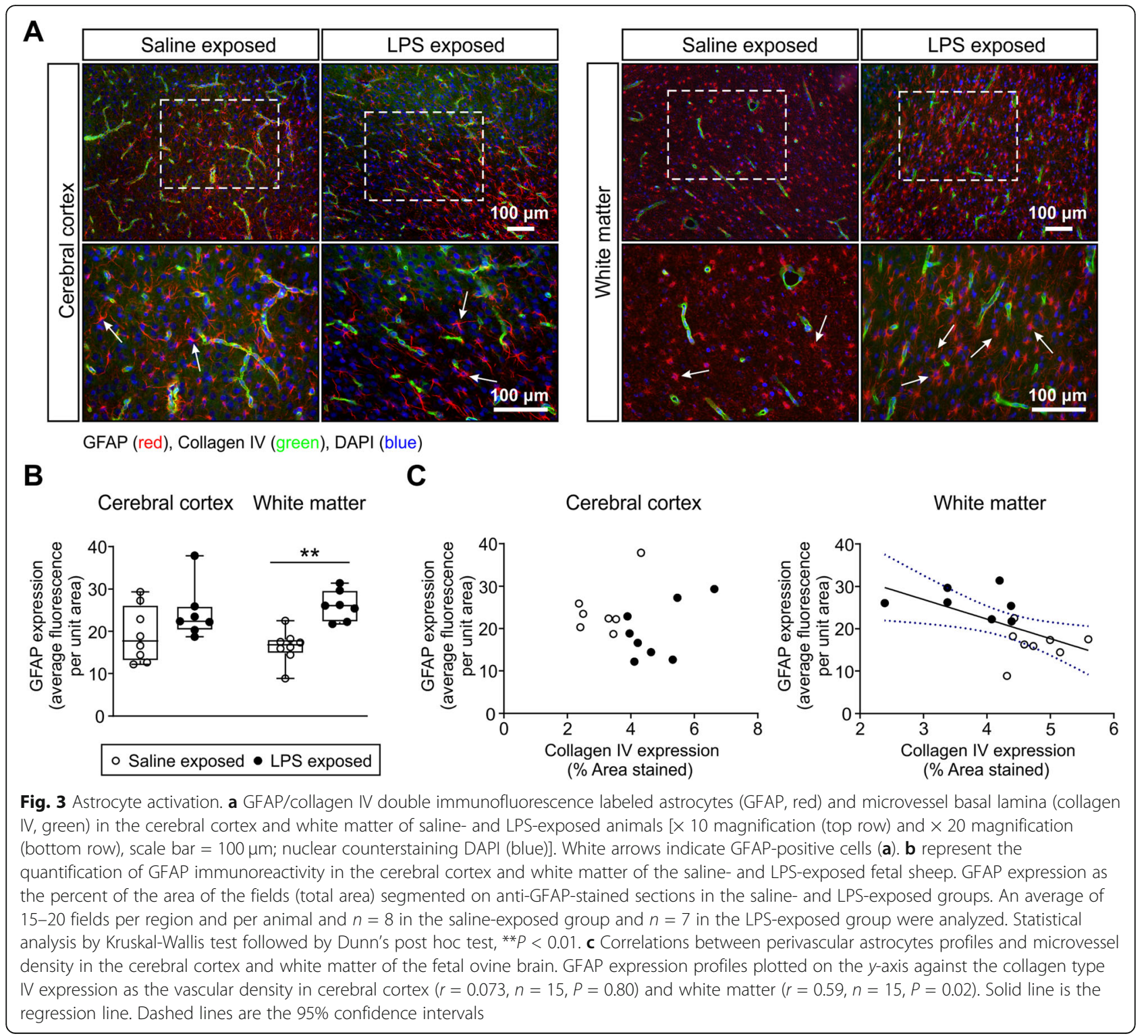

\section{Discussion}

Inflammation has been shown to be associated with impaired brain development and to predispose to neurodevelopmental abnormalities and disabilities in late childhood [46, 47]. The purpose of the current study was to test the hypothesis that inflammation adversely affects multiple components of the NVU in the cerebral cortex and white matter of the preterm ovine fetus after exposure to a prolonged inflammatory stimulus in utero. We demonstrate first that exposure to prolonged inflammation was associated with significant decreases in the microvascular density in the cerebral cortex and white matter of the ovine fetal brain 10 days after the onset of exposure to LPS. Secondly, pericyte microvascular coverage was decreased in the cerebral cortex and white matter. Thirdly, we observed increased astrogliosis, associated with decreases in the astrocyte end-feet coverage surrounding the microvasculature in the white matter.

This study examined fetal sheep at 103-104 days of gestation, which represents approximately $70 \%$ of the ovine gestation. The neuroanatomical development of the brain of the ovine fetus at this time in gestation is approximately similar to the preterm human brain between 28-32 weeks [36, 48, 49]. The neurodevelopment of the immature ovine brain is also similar to that of the premature infant with respect to the completion of neurogenesis, onset of cerebral sulcation, and emergence of the cortical components of the auditory and somatosensory evoked potentials [36, 50-53]. Consequently, studies of the vasculature in the brain of the preterm fetus at this time in gestation are relevant to understanding the development of brain injury in preterm infants. 


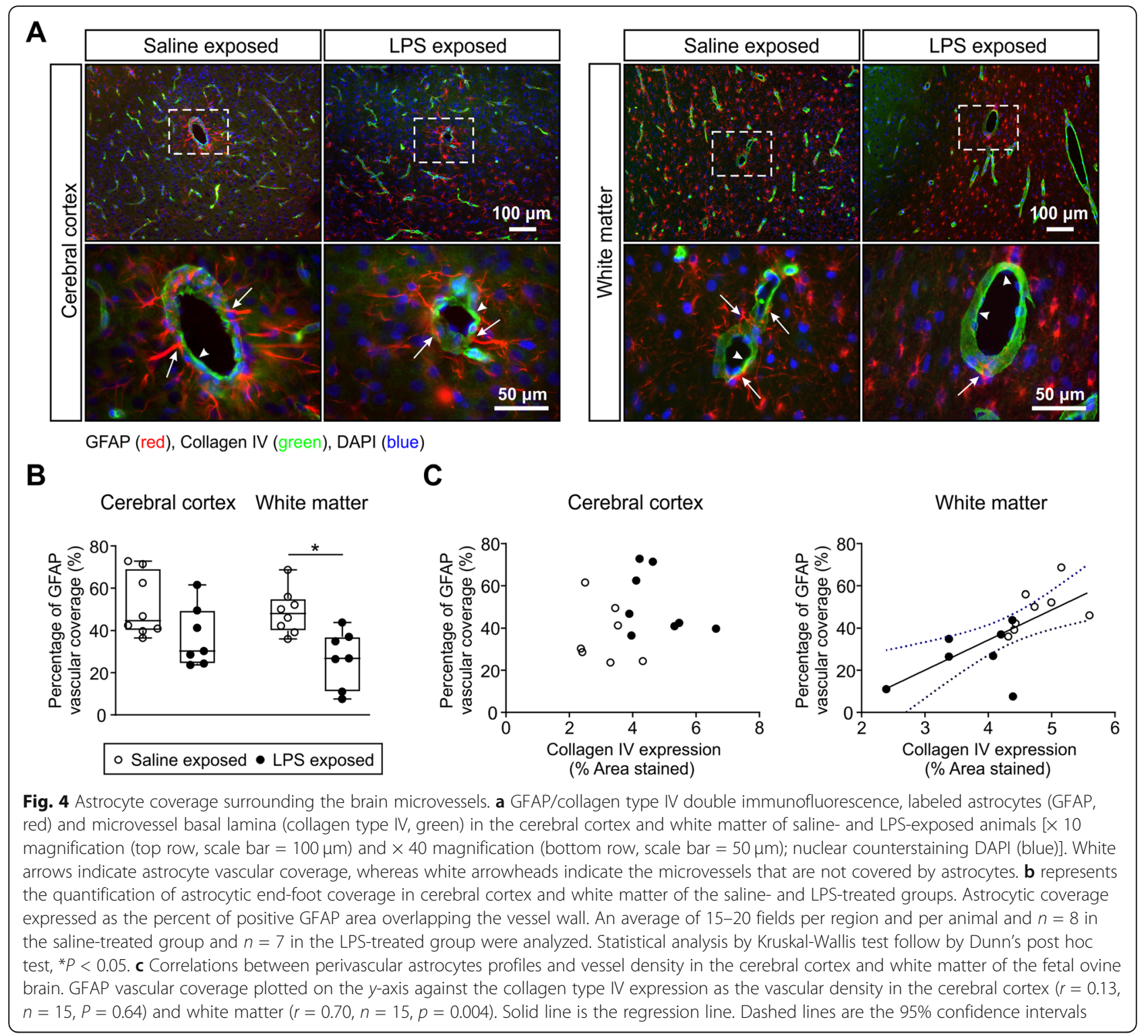

Treatment of neonatal rats with LPS has also been reported to result in white matter damage and acute transient disruption of the BBB [26]. In addition, LPS exposure has previously been suggested to increase BBB permeability in the ovine fetus [30,31]. These studies are consistent with findings suggesting that LPS-induced inflammation has important effects on the cerebral vasculature during fetal development [26, 30,31].

Inflammation of the neurovasculature is associated with activation of all cellular constituents in the NVU and with release of endogenous factors in brain cells. Therefore, we hypothesized that systemic inflammation could disrupt angiogenesis in the brain during development and alter the morphology of the NVU. Immunohistochemical analysis of the microvasculature after exposure of preterm fetal sheep to LPS in the present study demonstrated that the mean microvessel density was reduced in both the cerebral cortex and white matter. Vessel density was determined by measuring collagen type IV immunoreactivity. We have previously identified neovascularization within $72 \mathrm{~h}$ after the exposure of the fetal sheep brain to ischemia-reperfusion using the same methodology and marker [40]. Therefore, the immunohistochemical and morphometric analysis in this and our recent work suggests that collagen type IV is an excellent measure of microvessel density in the brain of the ovine fetus [40]. Collagen type IV is a major component of the basal lamina that provides structural stability to the vessel wall. Therefore, our findings suggest that prolonged exposure to LPS results in either a breakdown in the basal lamina and/or a reduction in vessel growth in the brain of the preterm fetus. 
Pericytes are critical cellular components of the NVU that are central to the development and maintenance of the $\mathrm{BBB}$ because of their contributions to the regulation of the endothelial cellular junctions [22, 54-56]. Pericytes participate in angiogenesis and perform cellular immune functions in addition to their contribution to the structural integrity and stability of the neurovasculature [56]. Coverage of the microvasculature by pericytes in the premature human cerebral cortex and white matter was approximately $90 \%$ using neural/glial antigen 2 (NG2) and platelet-derived growth factor receptor beta (PGDFR- $\beta$ ) as pericyte markers along with CD34 as an endothelial marker [57] compared to approximately 67 and $50 \%$ in the cortex and white matter, respectively, in control preterm fetal sheep in the present study. The lower pericyte coverage in the control preterm fetal sheep brain compared with the human brain could be related to species differences and/or pericyte marker differences and the use of CD34 as an endothelial marker versus collagen type IV as a basal lamina marker [57].

Exposure of the preterm fetal sheep to LPS resulted in significantly reduced pericyte coverage of the microvasculature in both the white matter and cerebral cortex. We speculate that the LPS-related reductions in pericyte coverage could result in vascular fragility, adverse consequences in BBB function, vascular contractility, and vessel growth and, consequently, alterations in brain perfusion and impaired brain growth and maturation. If similar exposure to inflammation to the preterm human fetus reduces pericyte coverage, this could in part contribute to the association between inflammation and increased risk of intraventricular hemorrhage and adverse neurological outcomes in premature infants [58, 59].

Astrocytes are essential to normal brain and cerebrovascular function [60]. The end-feet of astrocytes surround the brain microvasculature and regulate a wide variety of functions at the $\mathrm{BBB}$, and so astrocytes are key elements of the NVU [61]. Abnormalities in astrocyte end-feet can have dramatic consequences on the integrity of the $\mathrm{BBB}$ resulting in the development of brain disorders. Furthermore, pericytes in conjunction with astrocyte end-feet facilitate the expression of endothelial tight junction proteins, transporters, and enzymes within the microvasculature [17, 62, 63]. Astrocyte end-feet support ion transport, metabolite and energy substrate exchange from the blood to brain [61]. In addition, astrocytes secrete a variety of vasoactive molecules that modulate vascular tone [64-66]. Injury to gliovascular coupling could disrupt the integrity of the BBB and alter BBB transport function $[67,68]$. In the present study, inflammation induced by LPS exposure resulted in astrogliosis with typical end-feet swelling associated with a reactive morphology in both the cerebral cortex and white matter, with a significant overall increase in GFAP immunoreactivity in the white matter, consistent with previous reports $[14,35]$.

The coverage of astrocytes surrounding the brain microvessels in the control saline-exposed preterm fetal sheep was approximately $52 \%$ in the cerebral cortex and $49 \%$ in white matter. These findings are consistent with evidence in premature human infants at a similar stage of brain development using the same astroglial marker [69] supporting the contention that the neurovascular development of the preterm ovine fetal brain is similar to that of the preterm human brain between 28-32 weeks $[18,48,49]$. LPS exposure was associated with a reactive morphology of astrocytes and a significant decrease in the astrocytic end-foot coverage in the white matter. The loss of astrocytic and pericyte microvascular coverage after exposure to LPS and the consequent inflammatory stress-related condition suggests the potential for impaired gliovascular coupling that could adversely affect the BBB [30].

We have previously demonstrated that the proinflammatory cytokine IL-6 is increased in the blood and tumor necrosis factor- $\alpha$ (TNF- $\alpha)$ in the brain parenchyma of fetal sheep after exposure to LPS [14, 35]. Elevated cytokines within the brain parenchyma can originate locally from stimulated intrinsic cells and from infiltrated cells that originate in the systemic circulation and/or by crossing the BBB [70]. Microglia are the chief intrinsic contributors to the neuroinflammatory response among the intrinsic cellular elements in the brain [71, 72]. They populate the CNS very early in development, are present in all brain regions accounting for approximately $15 \%$ of glial cells [73], and contribute to synaptic remodeling, neuronal migration, and axonal growth during brain development [71, 72, 74, 75]. Microglia are also involved in angiogenesis and participate in NVU dynamics to maintain stability of the microenvironment and homeostasis of the brain parenchyma $[71,76]$.

Activated microglia migrate to the site of inflammation or of injury under inflammatory conditions to remove cellular debris and produce large quantities of proinflammatory mediators. We have previously reported and quantified infiltration of activated microglia into the periventricular white matter in LPS-exposed fetal sheep [14]. In our earlier report, the brains of the LPS-exposed fetal sheep showed patchy infiltration of activated microglia using isolectin B4 as the stain [14]. The numbers of microglia were significantly greater in the perivascular white matter compared with the control fetal sheep [14]. In the current study, we confirmed this observation by using Iba-1 as the microglia marker. Unfortunately, we were not able to quantify the microglia activation associated with the microvasculature because of technical issues related to the simultaneous staining of both the microvasculature and microglia in the sheep brain. 
The consequent inflammatory signals predispose to increases in BBB permeability and facilitate the entry of macrophages and cytokines from the blood into the brain to exacerbate neuronal damage. We used Iba-1 as a marker in this study because it recognizes the ramified cellular shape of the microglia. Resting type microglia were characterized in the saline control fetal sheep by a ramified phenotype evenly distributed in the perivascular space of the cerebral cortex and white matter (Supplemental Figure 1A and B upper panel). We confirmed the presence of activated amoeboid microglia in perivascular spaces in the white matter of LPS-exposed animals (Supplemental Figure 1B lower panel). By contrast, there were no morphological changes of microglia in the cerebral cortex of LPS-exposed fetal sheep (Supplemental Figure 1A lower panel).

The morphological alterations in the NVU in the cerebral cortex and white of preterm ovine brain after exposure to LPS in the present study likely contribute to inflammation-related brain injury. This postulate is supported by the close correlation between the responses of the various cellular components of the NVU and vessel density, further supporting the contribution of cellular communication in angiogenesis during development. Dysregulation in the components of the NVU could provide a therapeutic target to reduce the impact of inflammationrelated disorders in the preterm brain. However, the precise timing of such modulations and the ability of the NVU to recover remain to be determined in future experiments. Furthermore, it will be important to determine whether the changes in the NVU are transient or result in prolonged and more persistent damage to the NVU.

Although previous studies have demonstrated that acute and chronic inflammation results in robust inflammatory responses (glial activation and cytokine release) and cell death (neuronal apoptosis), this is the first report to demonstrate significantly reduced microvessels in the cerebral cortex and white matter of fetuses exposed to prolonged inflammation [7, 29, 77, 78]. This decrease in the amount of cerebral microvessels in the

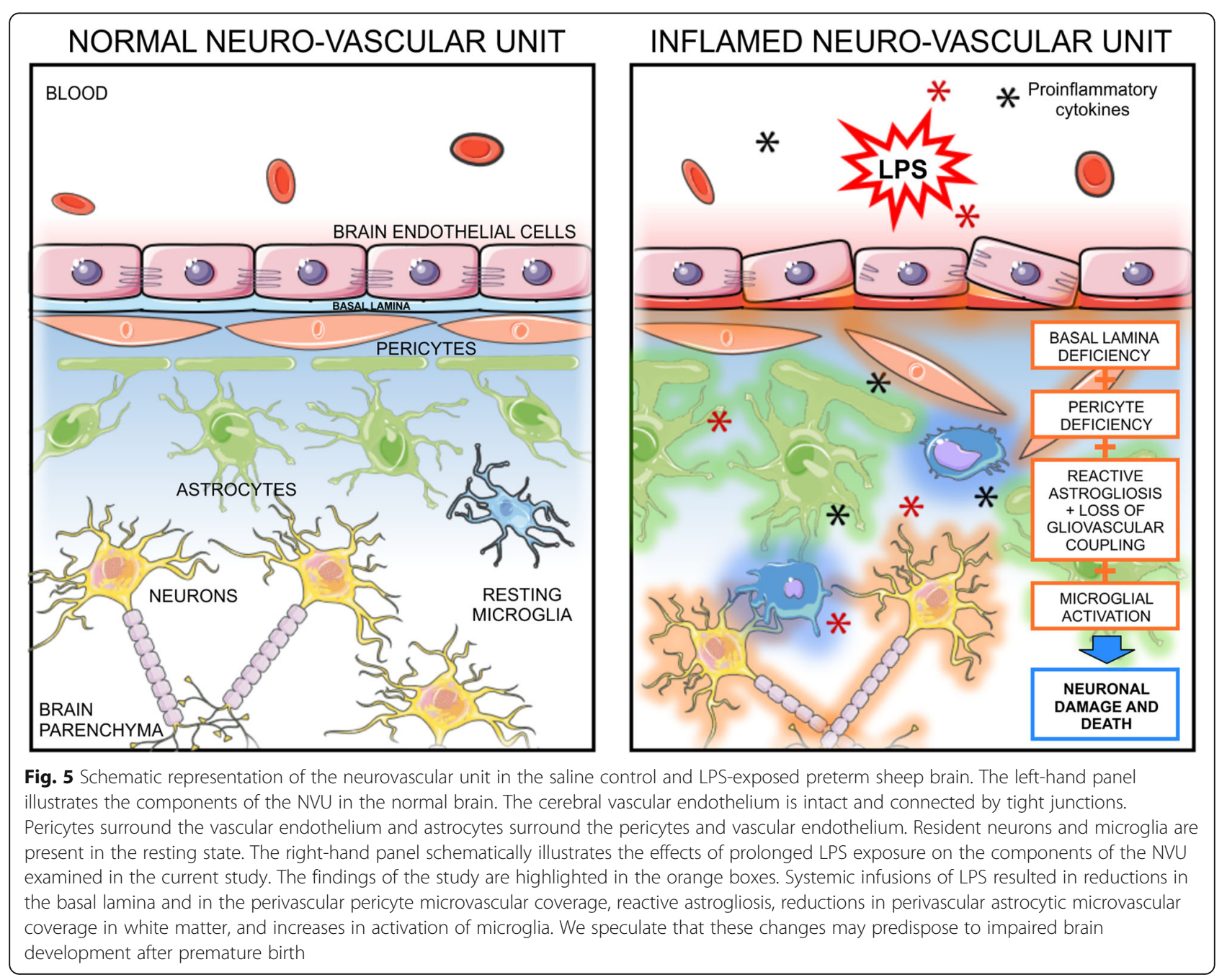


brain of fetuses exposed to LPS could have an important impact on brain development because the vasculature provides the brain with oxygen, glucose, and nutrition. Therefore, examination of treatment strategies to prevent or attenuate the changes that we observed would be important. Although antibiotics and antenatal corticosteroids are routinely used in pregnancies with premature rupture of membranes and chorioamnionitis, we do not know if these strategies or potential future anti-inflammatory strategies could attenuate or eliminate the changes that we observed in the neurovasculature of the fetal sheep after exposure to LPS [38, 79-83]. Even though some anti-inflammatory strategies have been shown to attenuate inflammation and changes in the BBB in animal models [83-86], there are as yet no clinically established antiinflammatory strategies to attenuate inflammatory changes in human pregnancies. Moreover, it remains to be determined whether treatment with antibiotics and/or anti-inflammatory strategies would reverse or attenuate the changes in the neurovasculature that we observed in the preterm fetal sheep.

\section{Conclusions}

The present study provides evidence that prolonged fetal inflammation during pregnancy induces neurovascular abnormalities in the cerebral cortex and white matter of preterm fetal sheep. The responses of the NVU to prolonged exposure to LPS in the preterm ovine fetus are schematically summarized in Fig. 5 . In the normal fetus (left panel), the cerebral vascular endothelium is intact and connected by tight junctions. Pericytes surround the vascular endothelium and astrocytes surround the pericytes and vascular endothelium. Resident neurons and microglia are present in the resting state. After LPS exposure (right-hand panel), there is decreased cerebral cortical and white matter vascular density, and astrogliosis, reduced pericyte and astrocytic microvascular coverage, and microglial activation. We speculate that these alterations in the NVU could predispose to neuronal injury and impaired brain growth and maturation $[7,14,15]$.

\section{Supplementary information}

Supplementary information accompanies this paper at https://doi.org/10. 1186/s12974-020-01852-y.

Additional file 1: Figure S1. Perivascular microglia activation. Representative images of Iba-1 staining (red) in the cerebral cortex (A) and white matter (B) of the saline (top row) and LPS (bottom low) exposed fetal sheep. $40 \times$ magnification, Scale bar $=20 \mu \mathrm{m}$, nuclear counterstaining DAPI (blue). White arrows indicate Iba-1 positive microglia. $\mathrm{VL}=$ vessel lumen

\section{Abbreviations}

LPS: Gram-negative endotoxin lipopolysaccharide; NVU: Neuro-vascular unit; BBB: Blood-brain barrier; GFAP: Glial fibrillary acidic protein; Iba-1: Ionized calcium-binding adaptor molecule 1

\section{Acknowledgements}

We gratefully acknowledge Virginia Hovanesian for her assistance with the immunohistochemical images.

\section{Authors' contributions}

$\mathrm{SD}, \mathrm{RG}, J \mathrm{OD}, \mathrm{CL}, \mathrm{AJG}$, and $\mathrm{LB}$ designed the study and performed in vivo experiments. AJG revised the manuscript. CD and FA performed immunofluorescence experiments and image analysis. XC prepared the figures for publication and revised the manuscript. CD analyzed the data, interpreted the results of the experiments, prepared the figures, and drafted the original manuscript version. BSS supervised the study and revised the manuscript. All authors reviewed the manuscript and agreed upon the final version.

\section{Funding}

Research reported in this publication was supported by the National Institute of General Medical Sciences of the National Institutes of Health under the following award numbers: Institutional Development Award (IDeA) from the National Institute of General Medical Sciences of the National Institutes of Health under grant number P30GM114750, National Institutes of Health (1R21NS095130 (BSS), 1R21NS096525 (BSS), 2R01HD057100 (BSS), and 2R44 NS084575 (BSS)), and the Health Research Council of New Zealand (17/601). The content is solely the responsibility of the authors and does not necessarily represent the official views of the National Institutes of Health or the Health Research Council of New Zealand.

\section{Availability of data and materials}

All data generated or analyzed for this manuscript during this study is contained within the manuscript.

\section{Ethics approval and consent to participate}

All procedures were approved by the Animal Ethics Committee of the University of Auckland, and carried out in accordance with the New Zealand Animal Welfare Act, and the Code of Ethical Conduct for animals in research established by the Ministry of Primary Industries, Government of New Zealand.

Consent for publication

Not applicable.

\section{Competing interests}

No conflicts of interest, financial or otherwise, are declared by the authors

\section{Author details}

'Department of Pediatrics, Women \& Infants Hospital of Rhode Island, Alpert Medical School of Brown University, 101 Dudley Street, Providence, RI 02905, USA. ${ }^{2}$ Department of Physiology, The University of Auckland, Auckland, New Zealand.

Received: 26 March 2020 Accepted: 21 May 2020

Published online: 28 May 2020

References

1. Bright HR, Babata K, Allred EN, Erdei C, Kuban KCK, Joseph RM, O'Shea TM, Leviton A, Dammann O, Investigators ES. Neurocognitive outcomes at 10 years of age in extremely preterm newborns with late-onset bacteremia. $J$ Pediatr. 2017;187:43-9 e41.

2. Leviton A, Allred EN, Dammann O, Engelke S, Fichorova RN, Hirtz D, Kuban KC, Ment LR, O'Shea TM, Paneth N, et al. Systemic inflammation, intraventricular hemorrhage, and white matter injury. J Child Neurol. 2013;28: 1637-45.

3. Leviton A, Joseph RM, Allred EN, Fichorova RN, O'Shea TM, Kuban KKC, Dammann $\mathrm{O}$. The risk of neurodevelopmental disorders at age 10 years associated with blood concentrations of interleukins 4 and 10 during the first postnatal month of children born extremely preterm. Cytokine. 2018; 110:181-8.

4. Harrison MS, Goldenberg RL. Global burden of prematurity. Semin Fetal Neonatal Med. 2016:21:74-9.

5. Patra A, Huang H, Bauer JA, Giannone PJ. Neurological consequences of systemic inflammation in the premature neonate. Neural Regen Res. 2017;12: 890-6. 
6. Hagberg $\mathrm{H}$, Mallard C. Effect of inflammation on central nervous system development and vulnerability. Curr Opin Neurol. 2005;18:117-23.

7. Bennet L, Dhillon S, Lear CA, Van den Heuij L, King V, Dean JM, Wassink G, Davidson JO, Gunn AJ. Chronic inflammation and impaired development of the preterm brain. J Reprod Immunol. 2018;125:45-55.

8. Volpe JJ. Brain injury in premature infants: a complex amalgam of destructive and developmental disturbances. Lancet Neurol. 2009;8:110-24.

9. Ortinau C, Neil J. The neuroanatomy of prematurity: normal brain development and the impact of preterm birth. Clin Anat. 2015;28:168-83.

10. Wang XY, Rousset Cl, Hagberg H, Mallard C. Lipopolysaccharide-induced inflammation and perinatal brain injury. Semin Fetal Neonatal Med. 2006;11:343-53.

11. Nitsos I, Rees SM, Duncan J, Kramer BW, Harding R, Newnham JP, Moss TJM. Chronic exposure to intra-amniotic lipopolysaccharide affects the ovine fetal brain. J Soc Gynecol Investig. 2006;13:239-47.

12. Gavilanes AWD, Strackx E, Kramer BW, Gantert M, van den Hove D, Steinbusch H, Garnier Y, Cornips E, Zimmermann L, Vles J. Chorioamnionitis induced by intraamniotic lipopolysaccharide resulted in an intervaldependent increase in central nervous system injury in the fetal sheep. Am J Obstet Gynecol. 2009;200.

13. Burd I, Balakrishnan B, Kannan S. Models of fetal brain injury, intrauterine inflammation, and preterm birth. Am J Reprod Immunol. 2012;67:287-95.

14. Mathai S, Booth LC, Davidson JO, Drury PP, Fraser M, Jensen EC, George S, Naylor A, Gunn AJ, Bennet L. Acute on chronic exposure to endotoxin in preterm fetal sheep. Am J Phys Regul Integr Comp Phys. 2013;304:R189-97.

15. Lear CA, Davidson JO, Booth LC, Wassink G, Galinsky R, Drury PP, Fraser M, Bennet L, Gunn AJ. Biphasic changes in fetal heart rate variability in preterm fetal sheep developing hypotension after acute on chronic lipopolysaccharide exposure. Am J Phys Regul Integr Comp Phys. 2014;307:R387-95.

16. ladecola C. The neurovascular unit coming of age: a journey through neurovascular coupling in health and disease. Neuron. 2017:96:17-42.

17. Abbott NJ, Friedman A. Overview and introduction: the blood-brain barrier in health and disease. Epilepsia. 2012;53:1-6.

18. Stonestreet BS, Patlak CS, Pettigrew KD, Reilly CB, Cserr HF. Ontogeny of blood-brain barrier function in ovine fetuses, lambs, and adults. Am J Phys Regul Integr Comp Phys. 1996;271:R1594-601.

19. Virgintino D, Errede M, Robertson D, Capobianco C, Girolamo F, Vimercati A, Bertossi M, Roncali L. Immunolocalization of tight junction proteins in the adult and developing human brain. Histochem Cell Biol. 2004;122:51-9.

20. Kniesel U, Risau W, Wolburg H. Development of blood-brain barrier tight junctions in the rat cortex. Dev Brain Res. 1996;96:229-40.

21. Ek CJ, Dziegielewska KM, Stolp H, Saunders NR. Functional effectiveness of the blood brain barrier to small water-soluble molecules in developing and adult opossum (Monodelphis domestica). J Comp Neurol. 2006;496:13-26.

22. Daneman R, Zhou L, Agalliu D, Cahoy JD, Kaushal A, Barres BA. The mouse blood-brain barrier transcriptome: a new resource for understanding the development and function of brain endothelial cells. PLoS One. 2010;5.

23. Saunders NR, Liddelow SA, Dziegielewska KM. Barrier mechanisms in the developing brain. Front Pharmacol. 2012;3.

24. Banks WA, Dohi K, Hansen K, Thompson HJ. Assessing blood granulocyte colony-stimulating factor as a potential biomarker of acute traumatic brain injury in mice and humans. Brain Behav Immun. 2016;52:81-7.

25. Disdier C, Stonestreet BS: Chapter 24 - Blood-brain barrier: effects of inflammatory stress. In Stress: Physiology, Biochemistry, and Pathology. Edited by Fink G: Academic Press; 2019: 325-336.

26. Stolp HB, Dziegielewska KM, Ek CJ, Potter AM, Saunders NR. Long-term changes in blood-brain barrier permeability and white matter following prolonged systemic inflammation in early development in the rat. Eur J Neurosci. 2005;22:2805-16.

27. Logsdon AF, Erickson MA, Chen X, Qiu J, Lim YP, Stonestreet BS, Banks WA: Inter-alpha inhibitor proteins attenuate lipopolysaccharide-induced bloodbrain barrier disruption and downregulate circulating interleukin 6 in mice. $J$ Cereb Blood Flow Metab 2019:271678X19859465.

28. Moretti R, Pansiot J, Bettati D, Strazielle N, Ghersi-Egea JF, Damante G, Fleiss B, Titomanlio L, Gressens P. Blood-brain barrier dysfunction in disorders of the developing brain. Front Neurosci. 2015;9.

29. Degos V, Favrais G, Kaindl AM, Peineau S, Guerrot AM, Verney C, Gressens P. Inflammation processes in perinatal brain damage. J Neural Transm. 2010; 117:1009-17.

30. Yan EW, Castillo-Melendez M, Nicholls T, Hirst J, Walker D. Cerebrovascular responses in the fetal sheep brain to low-dose endotoxin. Pediatr Res. 2004; 55:855-63.
31. Hutton LC, Castillo-Melendez M, Walker DW. Uteroplacental inflammation results in blood brain barrier breakdown, increased activated caspase 3 and lipid peroxidation in the late gestation ovine fetal cerebellum. Dev Neurosci. 2007:29:341-54.

32. Hong HK, Lee HJ, Ko JH, Park JH, Park JY, Choi CW, Yoon CH, Ahn SJ, Park KH, Woo SJ, Oh JY. Neonatal systemic inflammation in rats alters retinal vessel development and simulates pathologic features of retinopathy of prematurity. J Neuroinflammation. 2014;11.

33. Tremblay S, Miloudi K, Chaychi S, Favret S, Binet F, Polosa A, Lachapelle P, Chemtob S, Sapieha P. Systemic inflammation perturbs developmental retinal angiogenesis and neuroretinal function. Invest Ophthalmol Vis Sci. 2013:54:8125-39.

34. Lear CA, Davidson JO, Galinsky R, Yuill CA, Wassink G, Booth LC, Drury PP, Bennet L, Gunn AJ. Subclinical decelerations during developing hypotension in preterm fetal sheep after acute on chronic lipopolysaccharide exposure. Sci Rep. 2015;5.

35. van den Heuij LG, Mathai S, Davidson JO, Lear CA, Booth LC, Fraser M, Gunn AJ, Bennet L. Synergistic white matter protection with acute-on-chronic endotoxin and subsequent asphyxia in preterm fetal sheep. $J$ Neuroinflammation. 2014;11.

36. Back SA, Riddle A, Hohimer AR. Role of instrumented fetal sheep preparations in defining the pathogenesis of human periventricular whitematter injury. J Child Neurol. 2006;21:582-9.

37. Fowler JR, Simon LV: Chorioamnionitis. In StatPearls. Treasure Island (FL); 2020.

38. Sahni M, Franco-Fuenmayor ME, Shattuck K. Management of late preterm and term neonates exposed to maternal chorioamnionitis. BMC Pediatr. 2019;19:282

39. Cromey DW. Avoiding twisted pixels: ethical guidelines for the appropriate use and manipulation of scientific digital images. Sci Eng Ethics. 2010;16: 639-67.

40. Virgintino D, Girolamo F, Rizzi M, Ahmedli N, Sadowska GB, Stopa EG, Zhang J, Stonestreet BS. Ischemia/Reperfusion-induced neovascularization in the cerebral cortex of the ovine fetus. J Neuropathol Exp Neurol. 2014:73:495-506.

41. Smyth LCD, Rustenhoven J, Scotter EL, Schweder P, Faull RLM, Park TIH, Draqunow M. Markers for human brain pericytes and smooth muscle cells. $J$ Chem Neuroanat. 2018;92:48-60.

42. Abbott NJ. Inflammatory mediators and modulation of blood-brain barrier permeability. Cell Mol Neurobiol. 2000;20:131-47.

43. Allan SM, Rothwell NJ. Inflammation in central nervous system injury. Philos Trans R Soc Lond Ser B Biol Sci. 2003;358:1669-77.

44. Banks WA, Gray AM, Erickson MA, Salameh TS, Damodarasamy M, Sheibani N, Meabon JS, Wing EE, Morofuji Y, Cook DG, Reed MJ. Lipopolysaccharideinduced blood-brain barrier disruption: roles of cyclooxygenase, oxidative stress, neuroinflammation, and elements of the neurovascular unit. J Neuroinflammation. 2015;12.

45. Hagberg H, Mallard C, Ferriero DM, Vannucci SJ, Levison SW, Vexler ZS, Gressens $P$. The role of inflammation in perinatal brain injury. Nat Rev Neurol. 2015;11:192-208.

46. Green HF, Nolan YM. Inflammation and the developing brain: consequences for hippocampal neurogenesis and behavior. Neurosci Biobehav Rev. 2014;40: 20-34.

47. Hagberg H, Gressens P, Mallard C. Inflammation during fetal and neonatal life: Implications for neurologic and neuropsychiatric disease in children and adults. Ann Neurol. 2012;71:444-57.

48. Mclntosh GH, Baghurst KI, Potter BJ, Hetzel BS. Foetal brain development in the sheep. Neuropathol Appl Neurobiol. 1979;5:103-14.

49. Back SA, Riddle A, Dean J, Hohimer AR. The instrumented fetal sheep as a model of cerebral white matter injury in the premature infant. Neurotherapeutics. 2012;9:359-70.

50. Barlow RM. The foetal sheep: morphogenesis of the nervous system and histochemical aspects of myelination. J Comp Neurol. 1969;135:249-62.

51. Bernhard CG, Kolmodin GM, Meyerson BA. On the prenatal development of function and structure in the somesthetic cortex of the sheep. Prog Brain Res. 1967;26:60-77.

52. Cook CJ, Gluckman PD, Johnston BM, Williams C. The development of the somatosensory evoked potential in the unanaesthetized fetal sheep. J Dev Physiol. 1987:9:441-55.

53. Gunn AJ, Gunn TR, de Haan HH, Williams CE, Gluckman PD. Dramatic neuronal rescue with prolonged selective head cooling after ischemia in fetal lambs. J Clin Invest. 1997;99:248-56. 
54. Armulik A, Genove G, Mae M, Nisancioglu MH, Wallgard E, Niaudet C, He LQ, Norlin J, Lindblom P, Strittmatter K, et al. Pericytes regulate the bloodbrain barrier. Nature. 2010;468:557-U231.

55. Armulik A, Genove G, Betsholtz C. Pericytes: developmental, physiological, and pathological perspectives, problems, and promises. Dev Cell. 2011;21: 193-215.

56. Brown LS, Foster CG, Courtney JM, King NE, Howells DW, Sutherland BA. Pericytes and neurovascular function in the healthy and diseased brain. Front Cell Neurosci. 2019;13.

57. Braun A, Xu HM, Hu FR, Kocherlakota P, Siegel D, Chander P, Ungvari Z, Csiszar A, Nedergaard M, Ballabh P. Paucity of Pericytes in germinal matrix vasculature of premature infants. J Neurosci. 2007;27:12012-24.

58. Babnik J, Stucin-Gantar I, Kornhauser-Cerar L, Sinkovec J, Wraber B, Derganc M. Intrauterine inflammation and the onset of peri-intraventricular hemorrhage in premature infants. Biol Neonate. 2006:90:113-21.

59. Salafia CM, Minior VK, Rosenkrantz TS, Pezzullo JC, Popek EJ, Cusick W, Vintzileos AM. Maternal, placental, and neonatal associations with early germinal matrix/intraventricular hemorrhage in infants born before 32 weeks' gestation. Am J Perinatol. 1995;12:429-36.

60. Kimelberg HK, Nedergaard M. Functions of astrocytes and their potential as therapeutic targets. Neurotherapeutics. 2010;7:338-53.

61. Abbott NJ. Astrocyte-endothelial interactions and blood-brain barrier permeability. J Anat. 2002;200:629-38.

62. Wolburg H, Noell S, Mack A, Wolburg-Buchholz K, Fallier-Becker P. Brain endothelial cells and the glio-vascular complex. Cell Tissue Res. 2009;335:75-96.

63. Abbott NJ, Ronnback L, Hansson E. Astrocyte-endothelial interactions at the blood-brain barrier. Nat Rev Neurosci. 2006;7:41-53.

64. Attwell D, Buchan AM, Charpak S, Lauritzen M, MacVicar BA, Newman EA. Glial and neuronal control of brain blood flow. Nature. 2010;468:232-43.

65. Iadecola C, Nedergaard M. Glial regulation of the cerebral microvasculature. Nat Neurosci. 2007;10:1369-76.

66. Koehler RC, Roman RJ, Harder DR. Astrocytes and the regulation of cerebral blood flow. Trends Neurosci. 2009;32:160-9.

67. Abbott NJ. Blood-brain barrier structure and function and the challenges for CNS drug delivery. J Inherit Metab Dis. 2013:36:437-49.

68. Abbott NJ, Patabendige AA, Dolman DE, Yusof SR, Begley DJ. Structure and function of the blood-brain barrier. Neurobiol Dis. 2010;37:13-25.

69. El-Khoury N, Braun A, Hu FR, Pandey M, Nedergaard M, Lagamma EF, Ballabh P. Astrocyte end-feet in germinal matrix, cerebral cortex, and white matter in developing infants. Pediatr Res. 2006;59:673-9.

70. Sadowska GB, Chen XD, Zhang JY, Lim YP, Cummings EE, Makeyev O, Besio WG, Gaitanis J, Padbury JF, Banks WA, Stonestreet BS. Interleukin-1 beta transfer across the blood-brain barrier in the ovine fetus. J Cereb Blood Flow Metab. 2015;35:1388-95.

71. Mallard C, Tremblay ME, Vexler ZS. Microglia and neonatal brain injury. Neuroscience. 2019:405:68-76.

72. Tremblay ME, Stevens B, Sierra A, Wake H, Bessis A, Nimmerjahn A. The role of microglia in the healthy brain. J Neurosci. 2011;31:16064-9.

73. Nayak D, Roth TL, McGavern DB. Microglia development and function. Annu Rev Immunol. 2014;32:367-402.

74. Mosser CA, Baptista S, Arnoux I, Audinat E. Microglia in CNS development: Shaping the brain for the future. Prog Neurobiol. 2017;149-150:1-20.

75. Zhao XL, Eyo UB, Murugan M, Wu LJ. Microglial interactions with the neurovascular system in physiology and pathology. Dev Neurobiol. 2018;78:604-17

76. Thurgur $\mathrm{H}$, Pinteaux E. Microglia in the neurovascular unit: blood-brain barrier-microglia interactions after central nervous system disorders. Neuroscience. 2019;405:55-67.

77. Chew $L$, Takanohashi A, Bell M. Microglia and inflammation: impact on developmental brain injuries. Ment Retard Dev Disabil Res Rev. 2006;12:105-12.

78. Dammann O, Leviton A. Maternal intrauterine infection, cytokines, and brain damage in the preterm newborn. Pediatr Res. 1997:42:1-8.

79. Chatzakis C, Papatheodorou S, Sarafidis K, Dinas K, Makrydimas G, Sotiriadis A. Effect on perinatal outcome of prophylactic antibiotics in preterm prelabor rupture of membranes: network meta-analysis of randomized controlled trials. Ultrasound Obstet Gynecol. 2020;55:20-31.

80. Ryan M, Lacaze-Masmonteil T, Mohammad K. Neuroprotection from acute brain injury in preterm infants. Paediatr Child Health. 2019;24:276-90.

81. Yoon BH, Romero R, Park JY, Oh KJ, Lee J, Conde-Agudelo A, Hong JS. Antibiotic administration can eradicate intra-amniotic infection or intra- amniotic inflammation in a subset of patients with preterm labor and intact membranes. Am J Obstet Gynecol. 2019:221:142 e141-22.

82. Fowler JR, Simon LV: Chorioamnionitis. In StatPearls. Treasure Island (FL), 2019

83. Gomez-Lopez N, Romero R, Garcia-Flores V, Leng Y, Miller D, Hassan SS, Hsu CD, Panaitescu B. Inhibition of the NLRP3 inflammasome can prevent sterile intra-amniotic inflammation, preterm labor/birth, and adverse neonatal outcomes. Biol Reprod. 2019;100:1306-18.

84. Chen X, Sadowska GB, Zhang J, Kim JE, J Cummings EE, Bodge CA, Lim YP, Makeyev O, Besio WG, Gaitanis J, Threlkeld SW, Banks WA, Stonestreet BS. Neutralizing anti-interleukin-1 $\beta$ antibodies modulate fetal blood-brain barrier function after ischemia. Neurobiol Dis. 2015; 73:118-29.

85. Zhang JY, Sadowska GB, Chen XD, Park SY, Kim JE, Bodge CA, Cummings E, Lim YP, Makeyev O, Besio WG, et al. Anti-IL-6 neutralizing antibody modulates blood-brain barrier function in the ovine fetus. FASEB J. 2015;29: 1739-53.

86. Mottahedin A, Blondel S, Ek J, Leverin AL, Svedin P, Hagberg H, Mallard C, Ghersi-Egea JF, Strazielle N. N-acetylcysteine inhibits bacterial lipopeptidemediated neutrophil transmigration through the choroid plexus in the developing brain. Acta Neuropathol Commun. 2020;8:4.

\section{Publisher's Note}

Springer Nature remains neutral with regard to jurisdictional claims in published maps and institutional affiliations.
Ready to submit your research? Choose BMC and benefit from:

- fast, convenient online submission

- thorough peer review by experienced researchers in your field

- rapid publication on acceptance

- support for research data, including large and complex data types

- gold Open Access which fosters wider collaboration and increased citations

- maximum visibility for your research: over $100 \mathrm{M}$ website views per year

At $\mathrm{BMC}$, research is always in progress.

Learn more biomedcentral.com/submissions 